\title{
Properties and Performance of a New Compact HF Aerial Design for Multi-Band Operation
}

D. Telfer, J. Spencer

This work is an extension to that of Telfer and Austin [1] in that here a balanced feed embodiment of an inwardly-inclined folded dual monopole aerial is presented and discussed in terms of its improved performance over the original configuration. This includes greater control of the stability of the far-field (FF) lobe pattern with operating frequency, and a considerably extended frequency range (3:1 ratio) over which Near Vertical Incidence Skywave (NVIS) propagation in the high frequency (HF) bands can be exploited. Furthermore, the FF lobe patterns at frequencies $>2 \times$ the fundamental design frequency are such that advantage can be taken of conventional non-NVIS horizontal propagation at those frequencies using the same aerial. At the fundamental frequency, compactness of design and robustness of its NVIS FF pattern to orientation make the novel balanced aerial design a convenient replacement for a full-length low dipole in cluttered environments. The paper also presents a vehicle-mounted version for medium range operation within HF skip distances. Applications highlighted include stations for remote monitoring of environmental measurements in difficult or hostile terrain.

Keywords: aerial, antenna, multi-band, NVIS, HF, environmental monitoring.

\section{Notation/Abbreviations}

$\lambda \quad$ Wavelength (here expressed in $\mathrm{mm}$ ).

$\mathrm{dBi}$ The gain in decibels $(\mathrm{dB})$ relative to that of an isotropic radiator in free space.

HF High frequency. Refers to radio ,short wave' communication over the frequency range 3 to $30 \mathrm{MHz}$.

NVIS Near vertical incidence skywave. A high angle radio mode involving the ionosphere.

FF Far field pattern; refers to an envelope of constant field strength surrounding an energised radiating aerial. In conformity with the principle of reciprocity, the same envelope may be used to represent the profile of relative gain in various directions when using the aerial to receive radio signals.

DM Dual monopole, in this paper referring to aerials of a type consisting of inwardly inclined unfolded (i.e., single) elements on or near a ground plane. The elements cross over but do not physically touch.

SWR Standing wave ratio that would be on a transmission line if it were connected between a source of excitation and the aerial feed point. SWR of unity implies a perfect match between aerial and feeder cable.

\section{Introduction}

An overview of NVIS propagation with references has been given already in [1]. To summarise, there are three main modes of terrestrial HF radio propagation. These are: ground wave (GW in Fig. 1) for distances up to 50 miles but subject to blocking by hills, buildings and other obstacles. Secondly, over-the-horizon 'classical' skywaves, also launched horizontally, for intercontinental contacts employing skips between the ionosphere and the ground. And thirdly, NVIS launched at high angles (grey lines) and returned from the ionosphere, and having the useful property of straddling ground obstacles and overcoming the limitations of the dead zone in respect of the other two modes. This dead zone would otherwise extend from the groundwave maximum S1 to the nearest 'skip distance' from the ionosphere at S2. In the diagram, the NVIS range is represented by $R p(N)$ and varies with the state of the ionospheric layers E, F1 and E2. NVIS working is best at or just below the critical frequency, which during daytime is generally in the range 2 to $10 \mathrm{MHz}$ and commonly around $7 \mathrm{MHz}$. The aerials designs reported in this paper are for a fundamental resonant frequency of $7.05 \mathrm{MHz}$, the centre of the UK 7 to $7.1 \mathrm{MHz}$ amateur radio band. This is primarily for the convenience of practical testing by the author and similarly licensed persons. The application potential is far wider and includes emergency services and telemetry, e.g., environmental monitoring from locations in valleys or other difficult terrain, and at locations when and where links by line-of-sight VHF/UHF, satellite or mobile phone, may not be available. Under such circumstances, HF radio may be the only viable means of communication.

The FF pattern of an NVIS aerial should consist of a broad lobe directed upwards, with shapes ranging from near spherical (A in Fig. 1b) to an oblate spheroid (B), and with no horizontally directed sidelobes. This latter feature is particularly important for rejecting long distance 'skip' traffic when working local NVIS paths. Also, simultaneous reception of groundwave and NVIS signals can cause multi-path interference-induced fading. However, in some designs presented here, it is possible to use the same aerial for both NVIS work at or near the design frequency $f_{\mathrm{d}}$ (near vertical directivity), and also higher frequencies, e.g., around $2 \times f_{\mathrm{d}}$ or $3 \times f_{\mathrm{d}}$ with a different FF pattern, using horizontal lobes for over-the-horizon skip propagation.

We note that the numerical electromagnetic code (NEC) FF pattern computations take into account ground reflection of aerial geometry. In comparing FF patterns for this work, a perfect earth is assumed. As with any near-ground aerial system, the efficacy of the earth is affected by local conditions, which can cause losses and adversely affect aerial performance. The EZNEC ${ }^{\mathrm{TM}}$ software used is capable of simulating a range of imperfect earth scenarios, but these effects blur subtle FF pattern differences are not discussed further here. 


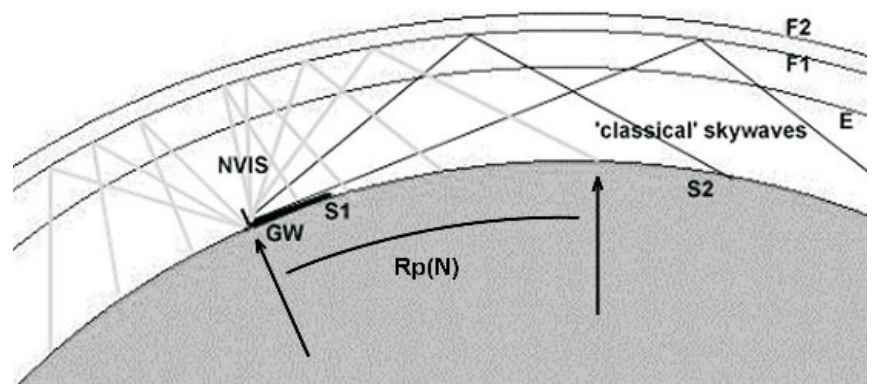

a)

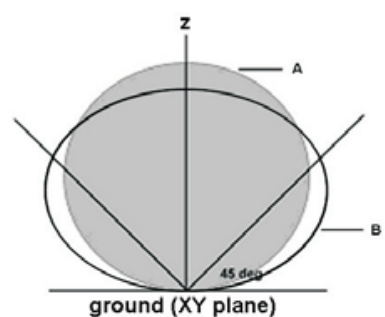

b)

Fig. 1: a) NVIS propagation (schematic), b) desired FF pattern

\section{Dual monopole aerial: unbalanced vs balanced configuration}

The main experimental aerial referred to in [1] was an unbalanced folded dual monopole (UBFDM) design (Fig. 2a) which, once computed, was relatively easy to set up and use.
However, the ability to maintain integrity of the near-spherical lobe pattern (Fig. 2c) over an extended frequency range either side of $f_{d}$ is limited. Simulations of this design also showed that the optimal FF pattern may not coincide with the condition of minimum SWR for good matching between aerial and feeder cable. One practical solution was to design the element configuration for optimal lobe shape, connect the

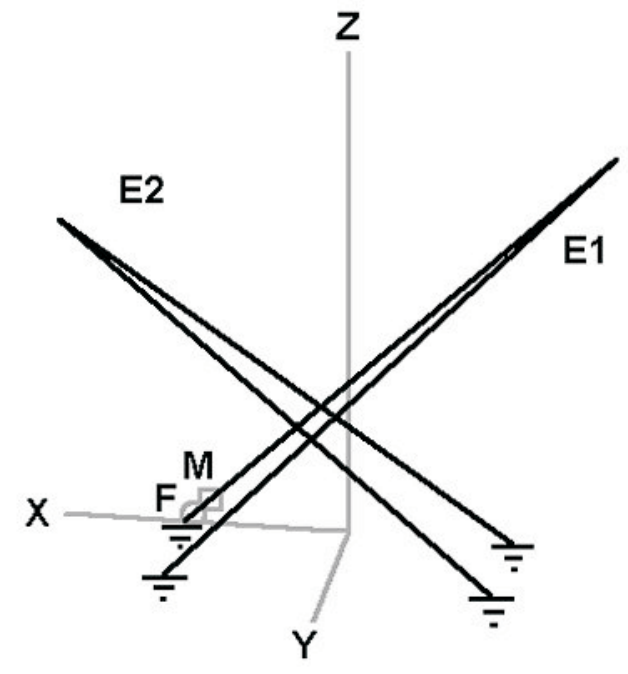

a)

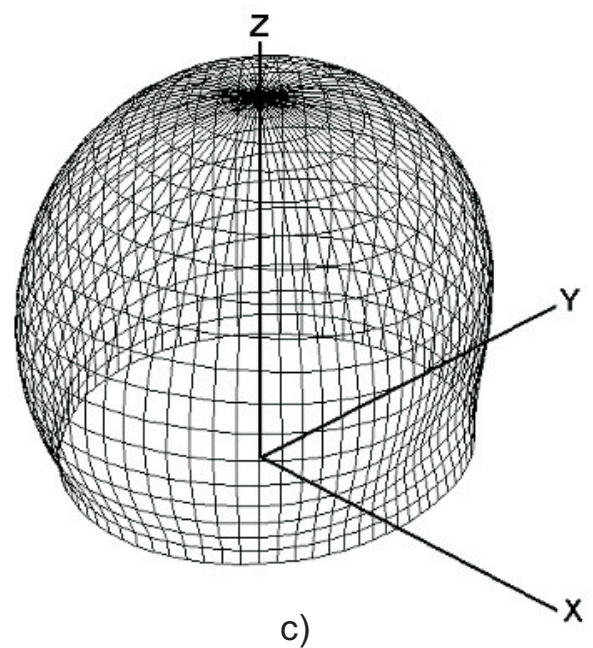

c)

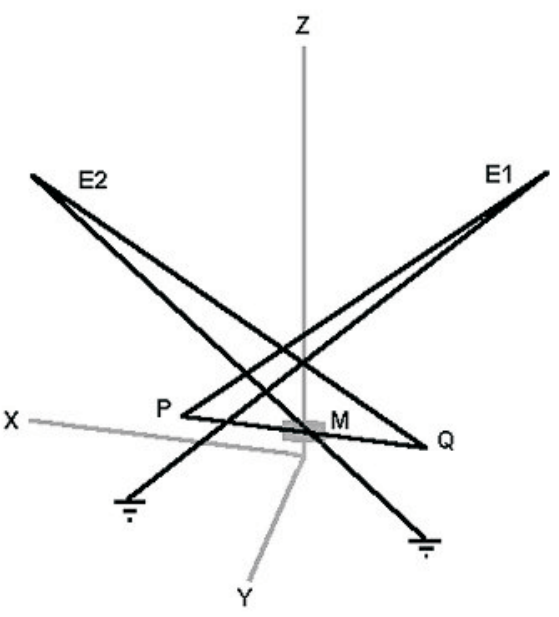

b)

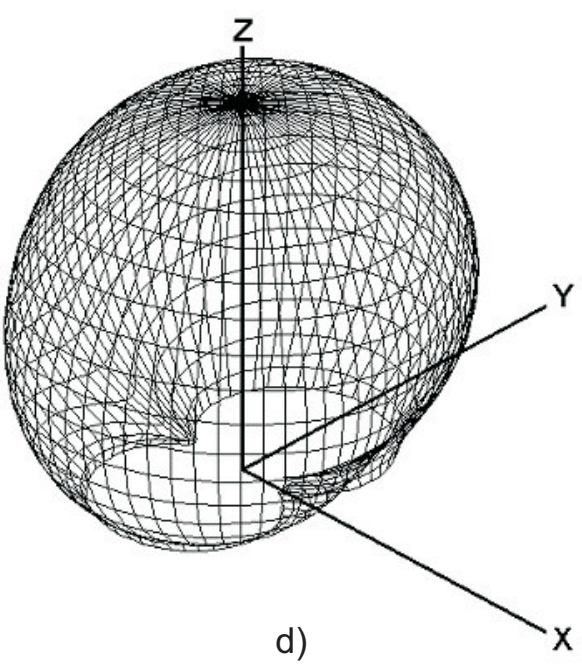

Fig. 2: a) unbalanced aerial: $\mathrm{M}$ is a matching reactance, close to the feed point $\mathrm{F}$, b) balanced aerial with integrated feed and matching unit M, c) UBDFM aerial $f_{\mathrm{d}}$ FF pattern, d) $f_{\mathrm{d}}$ FF lobe for BFDM aerial 
unbalanced (50 ohm co-axial) feeder directly to the aerial, then tune the system for minimal SWR at the transmitter end with an aerial tuning unit (ATU). This 'external tuning' worked well, but the challenge of obtaining an optimal lobe co-incident with perfect match at the aerial end remained. Therefore a 'matching unit' ( $\mathrm{M}$ in Fig. 2a) was introduced. This is a single reactance, either inductive for an electrically short aerial or capacitive for longer aerial elements.

When $\mathrm{M}$ is adjusted for resonance (minimum SWR) at the design frequency, attention can be switched to optimising the FF lobe, which is a property solely of the aerial configuration. This may be achieved by, e.g., making one element longer than the other, or changing the $x$-elevation of one element. Iteration of the whole process is carried out as required to give an acceptable lobe shape in the centre of the operational frequency range. But if the frequency range limitation (in our case $+/-0.2 \mathrm{MHz}$ at $7 \mathrm{MHz}$ ) for a well shaped NVIS lobe is not acceptable, the NVIS bandwidth may be extended by adopting balanced configurations. Two such solutions are explored in this work. One of these involved conversion of the foregoing UBFDM system to the balanced design of Fig. 2b. Although appearing more complex, both simulation and practical matching are made easier because the FF lobe retains its desired shape (Fig. 2d) over a much wider (cca 3:1) frequency range. The extra conductor length introduced between $\mathrm{P}$ and $\mathrm{Q}$ requires equal value pre-match capacitors at M, positioned each side of the feed point. The second solution is essentially the same matching arrangement driving a pair of non-folded elements.
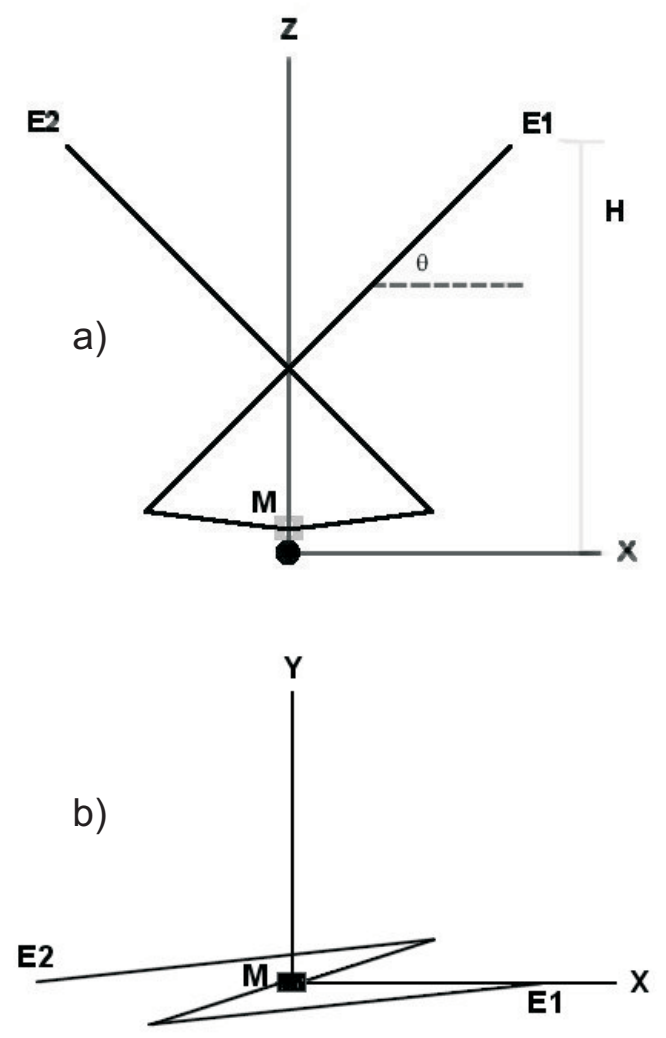

Fig. 3: a) simple DM aerial showing the x-elevation $\theta$ of elements $\mathrm{E} 1$ and $\mathrm{E} 2, \mathrm{~b}$ ) view of same aerial from $\mathrm{z}$ direction, showing inter-element gap

\section{Balanced dm aerials: effects of changes in $\mathbf{x}$-elevation}

Using non-folded elements (Fig. 3a) decreases the SWR bandwidth slightly, but a simplified balanced aerial, based on an embodiment in [2], facilitates modelling and testing, especially when changing element orientations.

EZNEC $^{\mathrm{TM}}$ software was employed to model the effects of changing x-elevation angle $\theta$. In order to unify scaling, the parameters $Z_{i}$ and $C_{m}$ (the matching capacitance values), are conveniently plotted against aerial height $\mathrm{H}$ (c.f., Fig. 3a), which is an important consideration in some locations.

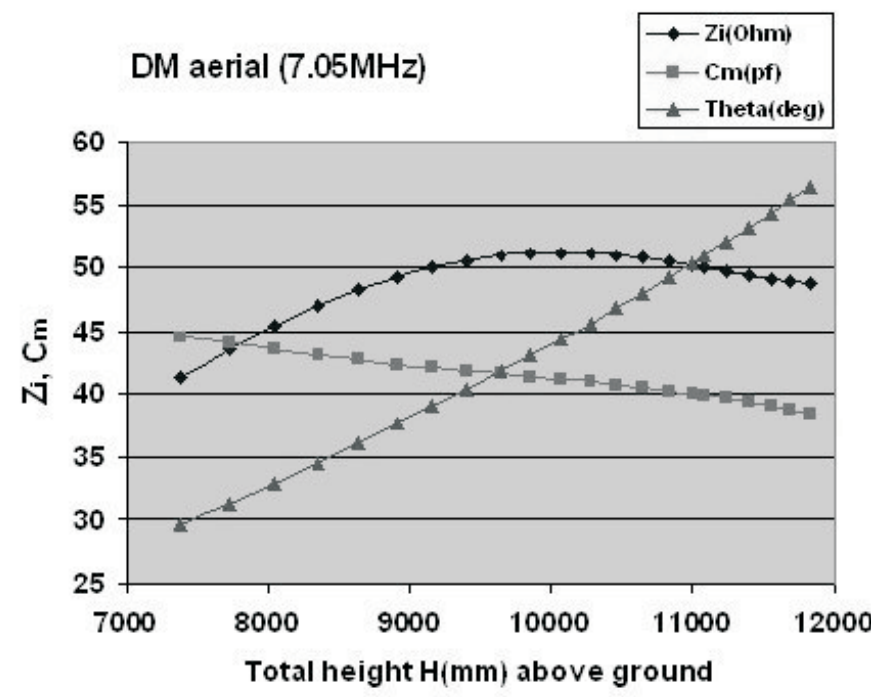

Fig. 4: Plots of $Z_{i}$ and $\theta$ in relation to total height (mm) for DM aerial with $f_{\mathrm{d}}=7.05 \mathrm{MHz}$ and concomitant adjustments to capacitor values $\mathrm{C}_{\mathrm{m}}(\mathrm{pF})$ in the matching unit $\mathrm{M}$ to make the feed impedance $Z_{i}(o h m)$ purely resistive. We note the considerable tolerance of $\mathrm{Z}_{\mathrm{i}}$ to $\theta$ with $\mathrm{Zi}(\max )$ at $\theta \sim 45$ degrees

Fig. 5a to $5 \mathrm{f}$ show how the angle $\theta$ affects the FF pattern. Fig. $5 \mathrm{~b}$ shows the FF pattern of a horizontal low dipole radiator for comparison.

The relative gain profile in Fig. 5a is shown in Fig. 5c for the XZ plane, for which the beamwidth for half-power points is 69.4 degrees. There is observable near-ground bifurcation along the $\mathrm{y}$-axis in Fig. 5a, and the YZ cross section is significantly broadened (Fig. 5 d) with a half-power point beamwidth of 99.4 degrees. Maximum gain (z-direction) is $8.26 \mathrm{dBi}$. By comparison, the dipole FF pattern of Fig. $5 \mathrm{~b}$ has half power beamwidths of 65 degrees in the XZ plane and 102.6 degrees in the $\mathrm{YZ}$ plane. In the dipole case, maximum (z-direction) gain is $8.35 \mathrm{dBi}$. Differences between the FF patterns for the $\mathrm{DM}$ aerial and a low $\lambda / 2$ dipole are very small and for practical purposes nearly identical. Physical dimensions of the test DM aerial (vertically aligned, $\theta=45$ degrees) are summarised as: lengths of E1, E2, $0.3051 \lambda$; length of centre wire containing $\mathrm{M}, 0.00706 \lambda$; length of wires joining the M-wire to lower ends of E1, E2, $0.07984 \lambda$. Heights (agl): M-wire, $0.0141 \lambda$; lower ends of E1, E2, $0.0235 \lambda$; upper ends of E1, E2, $0.2416 \lambda$. E1, E2 crossover gap $0.027 \lambda$.

Progressive variations in FF pattern with $\mathrm{q}$ are relatively small over the range 29.5 degrees (Fig. 5e) to 56.4 degrees (Fig. 5f). The most notable visual observations are the changes 
a)

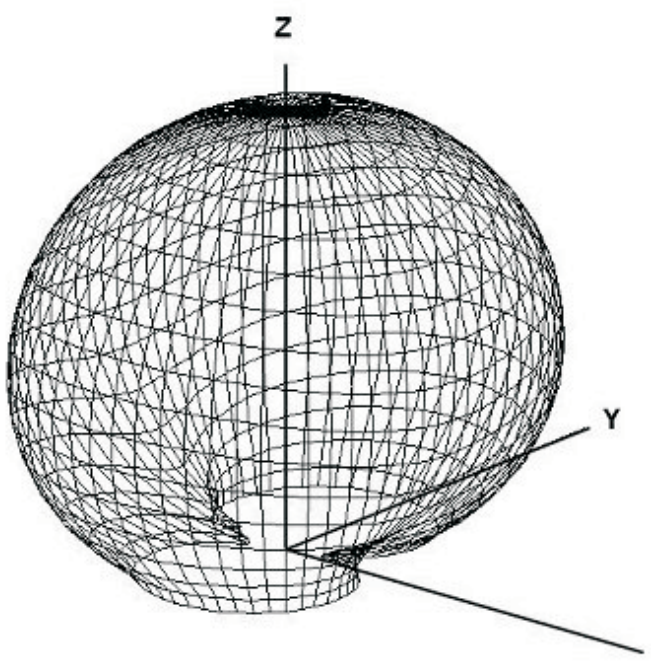

c)

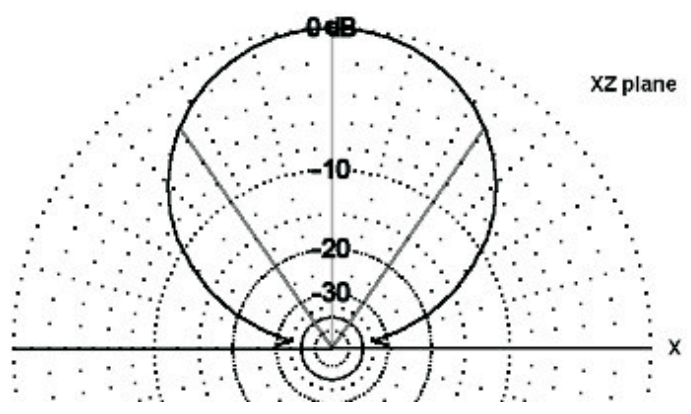

e)

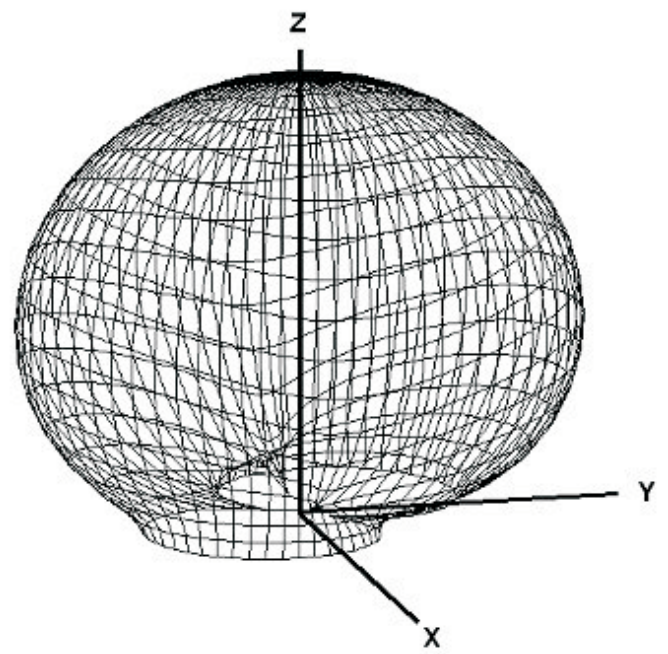

b)

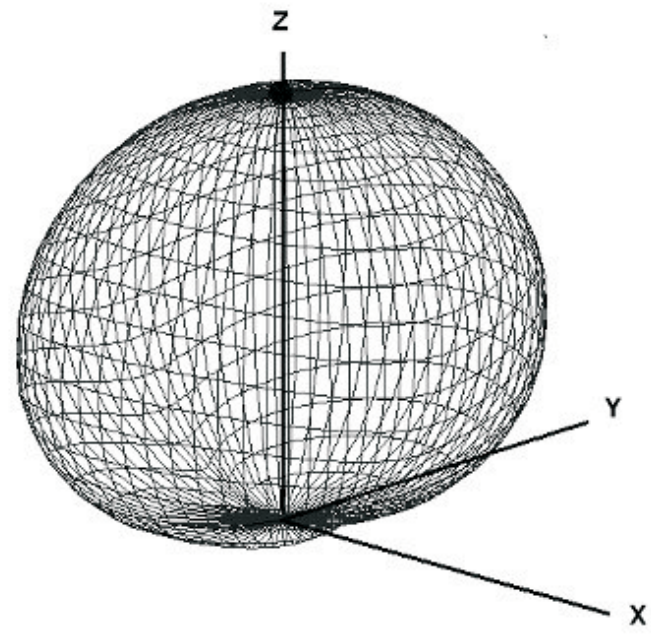

d)

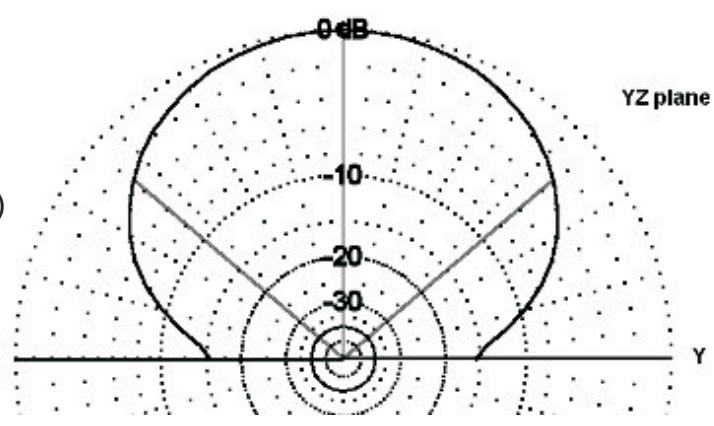

f)

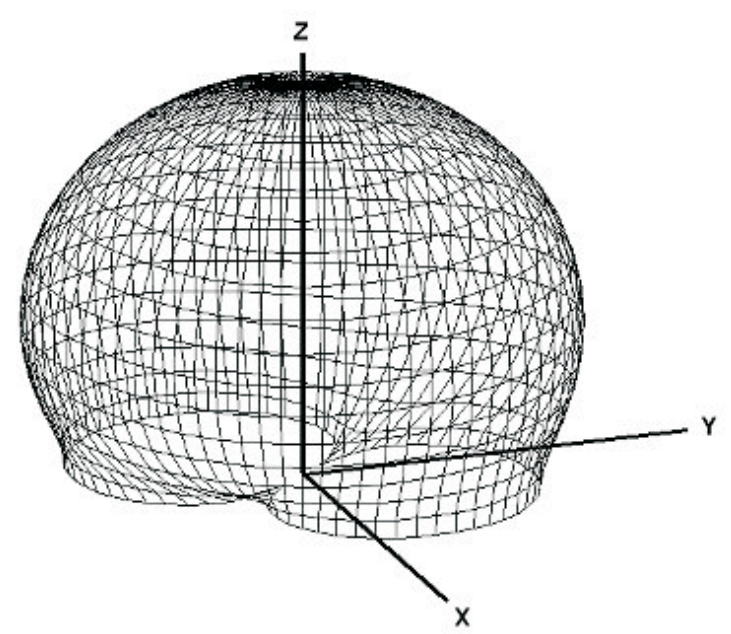

Fig. 5: a) FF pattern at $f_{\mathrm{d}}$ for DM aerial of Fig. 3a with $\theta=45$ degrees, b) FF pattern at $f_{\mathrm{d}}$ for fully extended low dipole (H=0.17 $\lambda$ ), c) XZ plane cross section of FF pattern in a), d) YZ plane cross section of FF pattern in b) showing broadening, e) FF pattern at $f_{\mathrm{d}}$ for DM aerial with x-elevation $\theta=29.5$ degrees, $\mathrm{f}$ ) FF pattern at $f_{\mathrm{d}}$ for DM aerial with x-elevation $\theta=56.4$ degrees

in structure and orientation of the near-ground bifurcations. As these are low-gain components of the profile, they make little practical difference, especially under 'real-earth' conditions, when distinctions between near-ground features are further reduced. The z-direction gains for the lobes in Figs. 5e and $5 \mathrm{f}$ are $8.78 \mathrm{dBi}$ and $7.08 \mathrm{dBi}$, respectively.

\section{Effects of changes in y-elevation}

In Fig. 6, the reference DM aerial has constant $\theta=45$ degrees, where $\theta$ is now the inclination of elements E1, E2 with respect to the medial axis, elevated $\varphi$ degrees in the y-direction. I.e., $(90-\varphi)$ is the tilt angle of the medial axis from the vertical (z) axis. The FF pattern in Fig. 7 for 58 degrees sideways tilt illustrates the robustness of the design to constraints in which sideways tilting is necessary, e.g., for obstacle avoidance or height restriction. However, in Fig. 8, $\mathrm{Z}_{\mathrm{i}}$ falls off increasingly with medial axis tilt (going right to left), whereas only small changes in matching capacitance $\mathrm{C}_{\mathrm{m}}$ are needed to maintain minimum SWR at $f_{\mathrm{d}}$. In practice, matching may be achieved using an ATU, e.g., at the transmitter end, but it is 


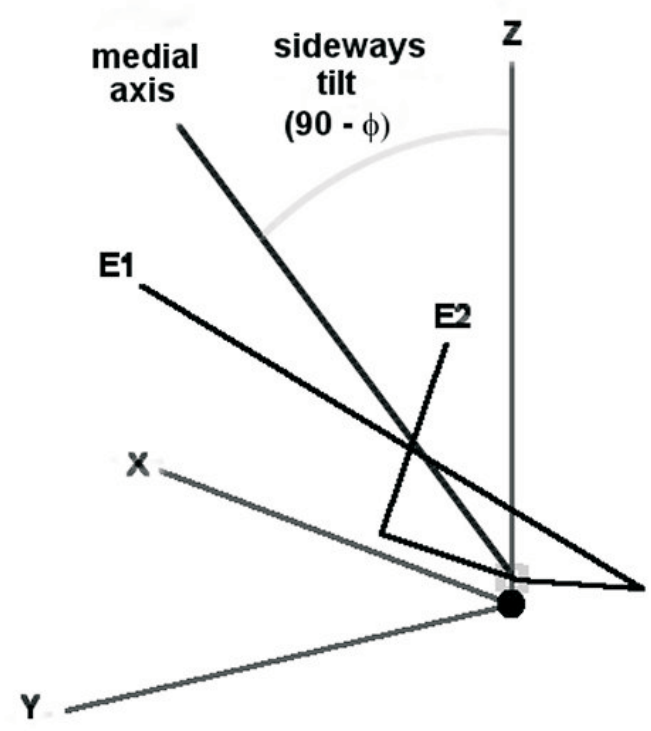

better to rely on a dedicated matching unit close to the aerial as indicated and then, if desired, use the ATU for 'tweaking'.

\section{The aerial matching unit}

A toroidal transformer (T1 in Figs. 9a,b) with suitable turns ratio matches the cable (generally $50 \mathrm{ohms}$ ) to $\mathrm{Z}_{\mathrm{i}}$. In practice, the secondary winding is connected to fixed pre-matching capacitors, each in parallel with a variable trimmer capacitor (see Fig. 9b) that is left preset after initial tuning. Any further tuning is done with the aerial tuning unit (ATU) connected at the transmitter end of the feeder cable.

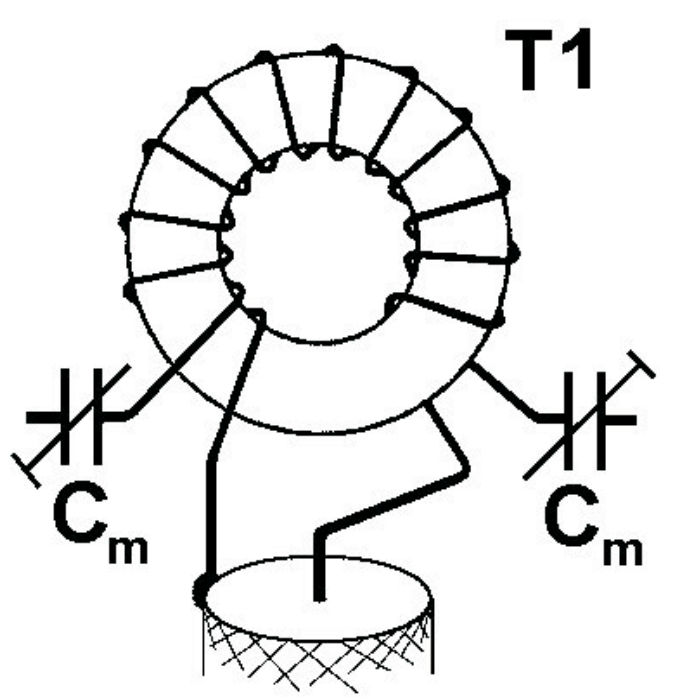

a)

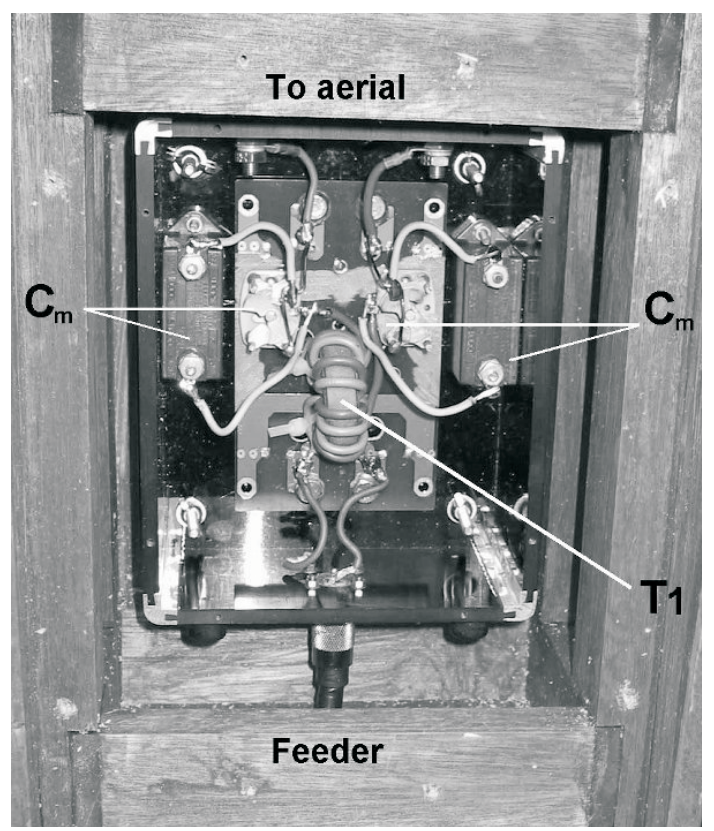

b)

Fig. 9: a) matching unit, schematic: primary feed connection to bifilar transformer $\mathrm{T} 1$ and secondary to the pre-matching capacitors $\mathrm{C}_{\mathrm{m}}$, b) matching unit construction at $7 \mathrm{MHz}$ and weatherproof housing

Fig. 8: Plots of $Z_{i}$ and $C_{m}$ against y-elevation $(\varphi)$ 


\section{SWR considerations and multiband properties}

The EZNEC ${ }^{\mathrm{TM}}$ predicted SWR at 7.0 to $7.1 \mathrm{MHz}$ for the DM aerial of Fig. 3 is shown below in Fig. 10a. An extended plot of SWR over the range 5 to $30 \mathrm{MHz}$ for the same aerial appears in Fig. 10b.

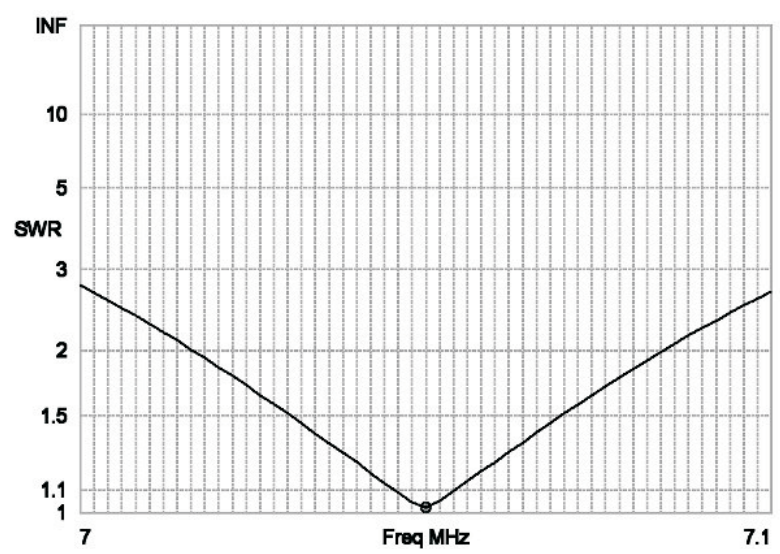

a)

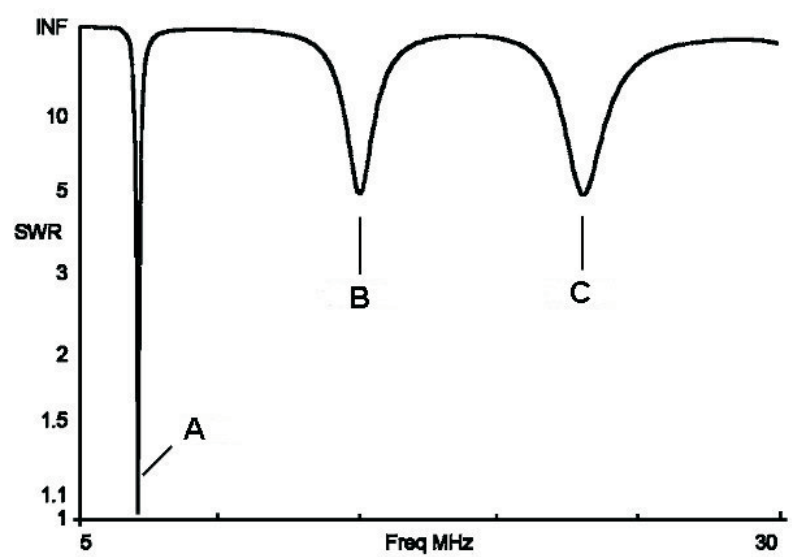

b)

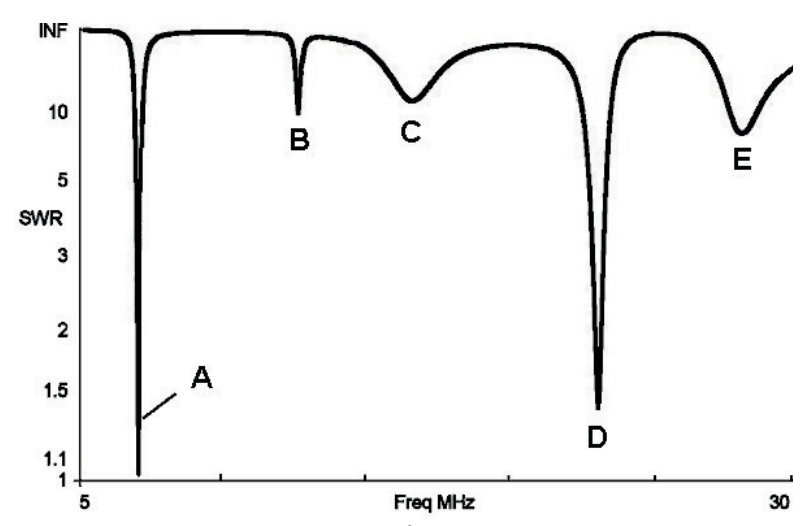

c)

Fig. 10: a) SWR plot, 7 to $7.1 \mathrm{MHz}$ for DM aerial of Fig. 3, $\mathrm{Z}_{\mathrm{i}}=50 \mathrm{ohms}$ at $7.05 \mathrm{MHz}$; b) SWR spectrum, $5 \mathrm{MHz}$ to $30 \mathrm{MHz}$ for the DM aerial of Fig. 3, for $Z_{i}=50$ ohms at $7.05 \mathrm{MHz}$; c) predicted SWR spectrum, range $5 \mathrm{MHz}$ to $30 \mathrm{MHz}$, of the BFDM aerial in Fig. $2 \mathrm{~b}$, for $\mathrm{Z}_{\mathrm{i}}=50 \mathrm{ohms}$ at $7.05 \mathrm{MHz}$, the occurrence of multiple peaks at higher frequencies (B to E) has positive implications for multiband operation
The plot of Fig. 10a covers the 40 metre UK amateur radio band (7.0 MHz to 7.1 MHz) which was used for practical tests. The SWR curve is such that only small adjustments to tuning with an aerial tuning unit (ATU) at the transmitter end of the cable will suffice for complete band coverage. This was borne out during practical NVIS experiments, where also support for the predicted aerial gain profile, as estimated from comparative signal strength readings with stations of known transmitter power and aerial configuration (e.g., horizontal dipole), was obtained. Similar in-band SWR and lobe properties were evident in experiments with the BFDM configuration of Fig. 2b, for which the 7.0 MHz to 7.1 MHz SWR plot was very similar. However, the extended spectral plot (Fig. 10c) revealed a more detailed signature, as anticipated from the increased aerial complexity. Positions of the higher
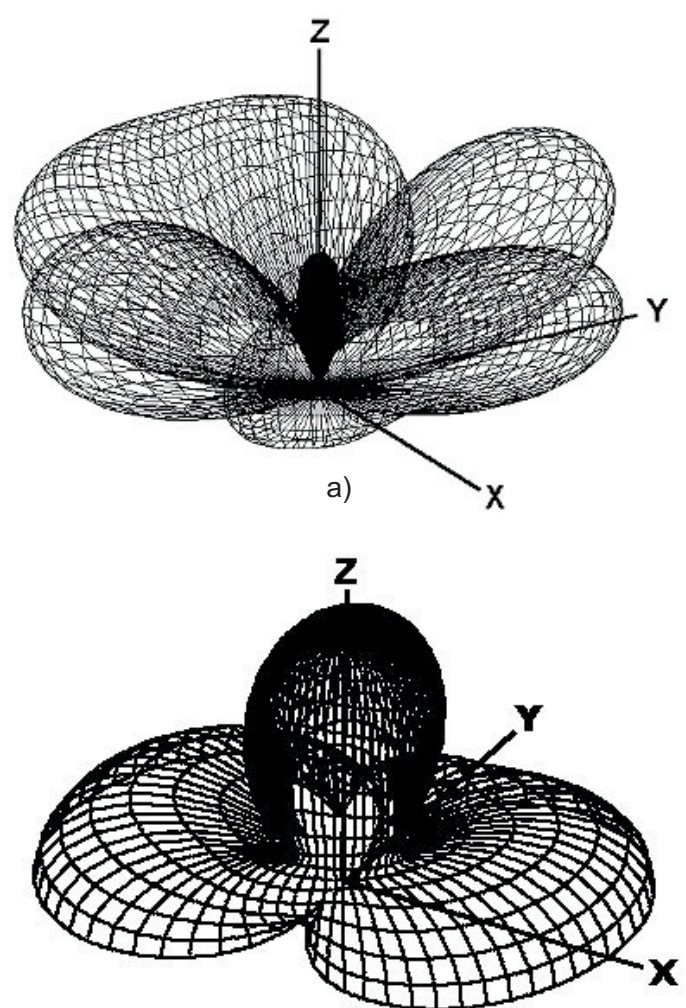

b)

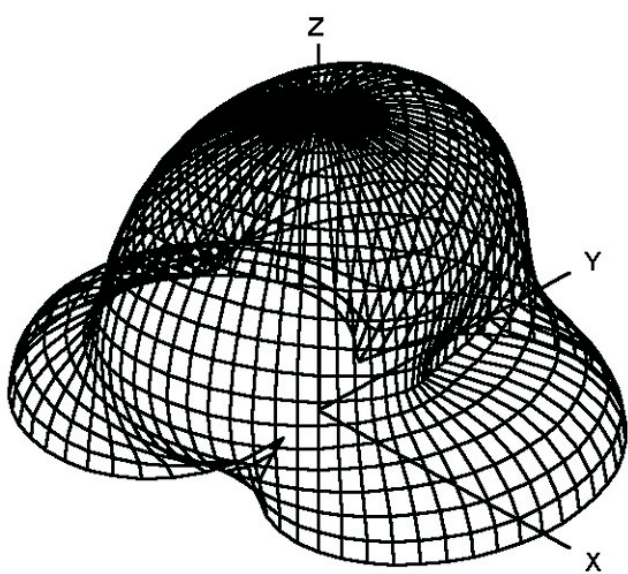

c)

Fig. 11: a) third harmonic FF pattern from a low dipole $(\mathrm{H}=0.17 \lambda), \mathrm{b})$ third harmonic FF pattern from the non-folded DM aerial of Fig. 3a, c) FF $f_{2}(14 \mathrm{MHz})$ plot for the BFDM aerial of Fig. $2 b$ 
frequency peaks $\mathrm{B}$ to $\mathrm{E}$ do not bear a simple relationship to $f_{\mathrm{d}}(\mathrm{A})$. Although aerial tuning at or near these peaks can be readily 'pulled' into a matched state at the transmitter with an ATU, high quality feeder cable should be used to minimise losses due to residual standing wave currents. This BFDM design has replaced the unbalanced one as the main HF aerial at the author's station and several months experience with has confirmed its similarity to a low dipole on the 7.0 $\mathrm{MHz}$ to $7.1 \mathrm{MHz}$ band.

In Fig. 10b, the simpler DM aerial spectrum is shown. Peaks A, B and C corresponding to the fundamental (design) frequency $f_{\mathrm{d}}\left(=f_{1}\right)$ and the approximate second and third harmonics, $f_{2}$ and $f_{3}$, respectively. It is well known that the dipole aerial can be most readily matched at $f_{1}$ and $f_{3}$. But how useful the DM aerial and its folded element variants would be for multiband operations depends on the higher frequency FF patterns, which, for working above NVIS frequencies, should have substantial horizontal lobes for 'skip' working.

Fig. 11a shows the FF pattern for the $7.05 \mathrm{MHz}$ low horizontal dipole operated near $f_{3}(21 \mathrm{MHz})$. This is compared in Fig. $11 \mathrm{~b}$ with the $f_{3} \mathrm{FF}$ pattern for the 'standard' DM aerial with $\mathrm{x}$-elevation $\theta=45$ degrees, and in with the BFDM aerial of Fig. 2b operated at $f_{2}$ (Fig. 11c) and $f_{3}$ (Fig. 2d).

The DM aerial $f_{3}$ FF pattern differs substantially from that of the low dipole, but has substantial energy input into sidelobes under 'perfect earth' conditions. However, under real-earth terrain conditions, these will be elevated and diminished in proportion to the losses. Although peaks B to E

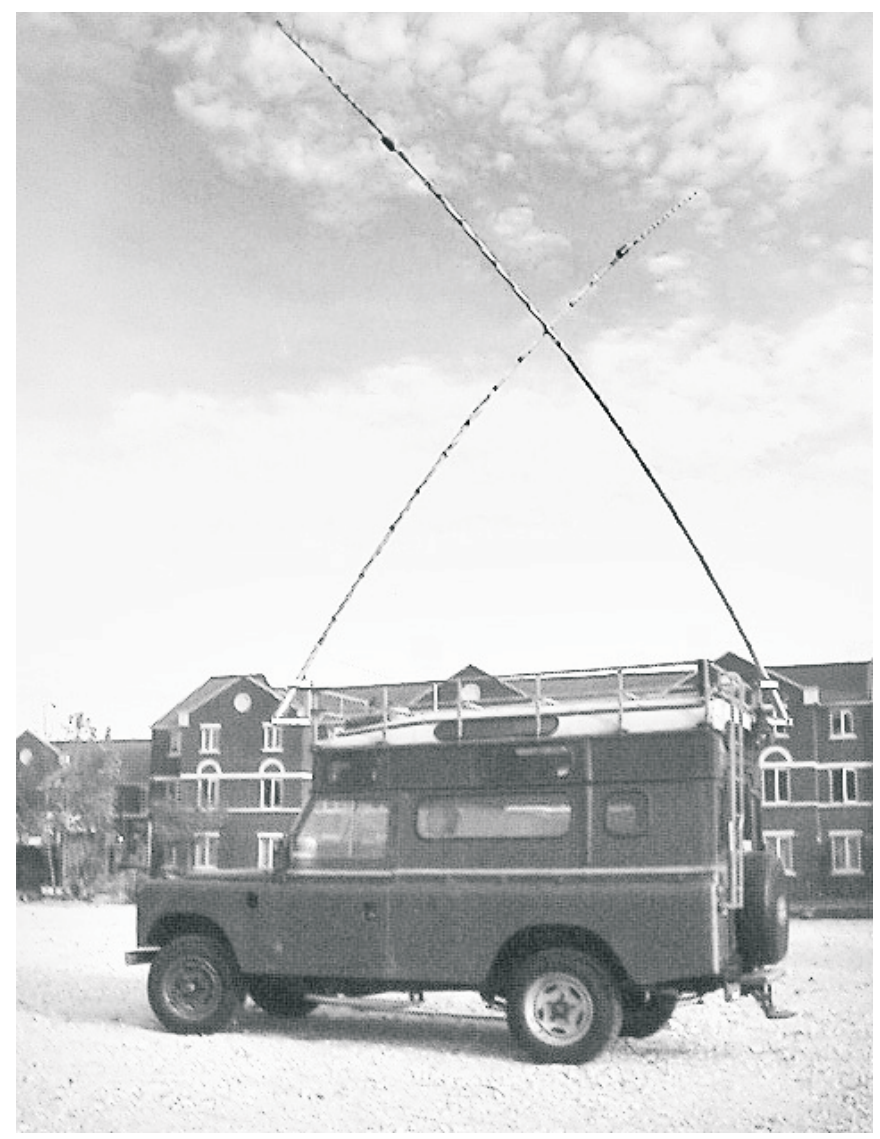

Fig. 12: Vehicular mounted shortened DM aerial, inductively loaded, has narrow SWR bandwidth but can be tuned readily with an ATU. Its potential for NVIS in fieldwork is being currently investigated in Fig. 10c for the BDFM aerial do not coincide exactly with $f_{2}$ and $f_{3}$, the $f_{2}$ plot is shown in Fig. $11 \mathrm{c}$, and the $f_{3}$ plot was very similar to Fig. $11 \mathrm{~b}$ for the DM aerial. Similar trends (not shown) were observed for the UBFDM aerial. These behaviours have also been confirmed by the author through radio skip working on $14 \mathrm{MHz}$ and $21 \mathrm{MHz}$ bands.

\section{Conclusions}

Computer simulation and practical testing of a number of dual inwardly-inclined monopole aerials for NVIS has confirmed the viability of this design avenue. In particular, the balanced feed variants exhibit consistency of vertical far-field pattern shape with frequency and element orientation, the latter augmenting spatial adaptability and compactness without compromising NVIS performance. Versatility is further exemplified by useful horizontal gain achieved at frequencies above those used for NVIS propagation, allowing the same aerial to be used, without further adjustment, for multi-band operation. Potential for applications in telemetry and emergency communications from shielded locations and difficult terrain is thereby demonstrated.

\section{Acknowledgments}

Thanks are due to our colleague Dr. Brian Austin for his continuing interest and many fruitful discussions, and to Dr. Alan Brown of the Countryside Council for Wales for insights into telemetry applications.

\section{References}

[1] Telfer, D. J., Austin, B. A.: "Novel Antenna Design for Near-Vertical Incidence (NVIS) HF Communications." Proc. $2^{\text {nd }}$ International Conference on Advanced Engineering Design [Electrical Engineering Section]. University of Glasgow, 2001, June 24-26.

[2] Telfer, D. J.: "Multiple Monopole Aerial." UK Patent GB2375235. April 2004.

\section{Dr. Duncan Telfer \\ e-mail: djtelfer@liverpool.ac.uk}

\section{Dr. Joe Spencer}

Centre for Intelligent Monitoring Systems

University of Liverpool

Department of Electrical Engineering and Electronics Brownlow Hill

Liverpool L69 3GJ 